\title{
Dynamics of circulating hypoxia-mediated miRNAs and tumor response in patients with high-grade glioma treated with bevacizumab
}

\author{
*Tali Siegal, MD, ${ }^{1,2}$ Hanna Charbit, MSc, ${ }^{2}$ Iddo Paldor, MD, ${ }^{3}$ Bracha Zelikovitch, ${ }^{2}$ \\ Tamar Canello, PhD, ${ }^{2}$ Arriel Benis, PhD, ${ }^{2}$ Michael L. Wong, PhD, FRACS ${ }^{4}$ \\ Andrew P. Morokoff, MBBS, PhD, FRACS, ${ }^{4,5}$ Andrew H. Kaye, MD, FRACS, ${ }^{4,5}$ and Iris Lavon, PhD² \\ ${ }^{1}$ Center for Neuro-Oncology, Davidoff Institute of Oncology, Rabin Medical Center, Campus Beilinson, Petach Tikva; ${ }^{2}$ Leslie and \\ Michael Gaffin Center for Neuro-Oncology and Department of Neurology, The Agnes Ginges Center for Human Neurogenetics; \\ ${ }^{3}$ Department of Neurosurgery, Hadassah Hebrew University Medical Center, Jerusalem, Israel; and Departments of \\ ${ }^{4}$ Neurosurgery and ${ }^{5}$ Surgery, The Royal Melbourne Hospital and The University of Melbourne, Australia
}

OBJECTIVE Bevacizumab is an antiangiogenic agent under investigation for use in patients with high-grade glioma. It produces a high rate of radiological response; however, this response should be interpreted with caution because it may reflect normalization of the tumor vasculature and not necessarily a true antitumor effect. The authors previously demonstrated that 4 hypoxia-mediated microRNAs (miRNA)-miR-210, miR-21, miR-10b, and miR-196b-are upregulated in glioma as compared with normal brain tissue. The authors hypothesized that the regulation and expression of these miRNAs would be altered in response to bevacizumab treatment. The object of this study was to perform longitudinal monitoring of circulating miRNA levels in patients undergoing bevacizumab treatment and to correlate it with tumor response.

METHODS A total of 120 serum samples from 28 patients with high-grade glioma were prospectively collected prior to bevacizumab $(n=15)$ or temozolomide (TMZ; $n=13$ ) treatment and then longitudinally during treatment. Quantification of the 4 miRNAs was evaluated by real-time polymerase chain reaction using total RNA extracted from the serum. At each time point, tumor response was assessed by Response Assessment in Neuro-Oncology criteria and by performing MRI using fluid attenuated inversion recovery (FLAIR) and contrast-enhanced images.

RESULTS As compared with pretreatment levels, high levels of miR-10b and miR-21 were observed in the majority of patients throughout the bevacizumab treatment period. miR-10b and miR-21 levels correlated negatively and significantly with changes in enhancing tumor diameters $(r=-0.648, p<0.0001)$ in the bevacizumab group but not in the TMZ group. FLAIR images and the RANO assessment did not correlate with the sum quantification of these miRNAs in either group. CONCLUSIONS Circulating levels of miR-10b and miR-21 probably reflect the antiangiogenic effect of therapy, but their role as biomarkers for tumor response remains uncertain and requires further investigation.

http://thejns.org/doi/abs/10.3171/2015.8.JNS15437

KEY WORDS high-grade glioma; glioblastoma; microRNA; bevacizumab; antiangiogenic therapy; temozolomide; circulating biomarkers; oncology

$\mathrm{T}$ Herapeutic modalities for glioblastoma multiforme (GBM) include resection, radiation, chemotherapy, and recently antiangiogenic therapy such as bevacizumab, which targets vascular endothelial growth factor (VEGF). Antiangiogenic therapy can produce an early, marked decrease in contrast enhancement during imaging studies and consequently reads as a high rate of radiologi- cal response. . $^{416}$ However, these radiological responses should be interpreted with caution because they are partly the result of normalization of the tumor vasculature and not always necessarily indicative of a true antitumor effect. Moreover, antiangiogenic therapy can increase the tendency of tumor cells to coopt existing blood vessels, resulting in an invasive, nonenhancing growth pheno-

ABBREVIATIONS FLAIR = fluid attenuated inversion recovery; GBM = glioblastoma multiforme; HIF-1 = hypoxia-inducible factor-1; miRNA = microRNA; PCR = polymerase chain reaction; RANO = Response Assessment in Neuro-Oncology; $R Q=$ relative quantification; $T M Z=$ temozolomide; VEGF = vascular endothelial growth factor. SUBMITTED February 25, 2015. ACCEPTED August 13, 2015.

INCLUDE WHEN CITING Published online January 22, 2016; DOI: 10.3171/2015.8.JNS15437.

* Drs. Siegal, Kaye, and Lavon and Ms. Charbit contributed equally to this work 
type. ${ }^{5,24,30}$ Progressive nonenhancing tumor can sometimes be detected by a continuous increase in T2 or fluid attenuated inversion recovery (FLAIR) signals on MRI, which are suggestive of infiltrative tumor. The presence of progressive nonenhancing tumor is often associated with clinical deterioration that determines the true course of the disease, despite the absence of an enhancing mass. The multiple facets of clinical and radiological appearance create circumstances that are defined as pseudoprogression or pseudoresponse. Standardized response criteria for clinical trials were therefore needed; the Response Assessment in Neuro-Oncology (RANO) Working Group developed such new criteria for brain tumors that assemble radiological and clinical parameters into 4 categories of response. ${ }^{36}$

Reliable and robust biomarkers might detect the presence of tumor, tumor activity, and response to treatment and could serve as an essential addition to the current diagnostic arsenal. Unfortunately, there are no biomarkers that can help monitor the response of GBM to therapy, and consequently, tumor status is evaluated only by clinical parameters standardized by the RANO criteria. Measurable biomarkers that could be detected in the bloodstream would have a considerable advantage because of their relatively easy accessibility, which facilitates repetitive assessments. To date, diverse categories of tumor-associated circulating biomarkers have been investigated, ${ }^{17}$ including circulating nucleic acids (DNA, RNA, and microRNA [miRNA]). We previously demonstrated that cell-free circulating DNA in patients with glial tumors can be used to identify genetic and epigenetic alterations that are present in the tumor tissue. ${ }^{18}$ In another study, we investigated the profile of miRNA expression in glial tumors and identified miRNAs that were upregulated in gliomas as compared with normal brain tissue. ${ }^{19}$ We selected 6 miRNAs with the highest expression levels (miR-10b, miR-196b, miR-210, miR-302, miR-1271, and miR-21) and evaluated their presence in the serum of patients harboring GBM. Four of the selected miRNAs are reported to be hypoxia-mediated miRNAs (miR-210, miR-21, miR-10b, and miR-196b). ${ }^{31}$ The regulation of these and other hypoxia-mediated miRNAs either is dependent on hypoxiainducible factor-1 (HIF-1) or is HIF-1-independent. ${ }^{31}$ It is therefore likely that both the regulation and expression of these miRNAs will be altered by antiangiogenic therapy. However, it is not clear in what way they will be altered because antiangiogenic agents can induce paradoxical effects on tumor vasculature; it is possible to observe both vascular normalization that improves nutrient and oxygen transport, and vessel pruning that reduces oxygenation and increases hypoxia..$^{153}$ In this study we tested the hypothesis that antiangiogenic treatment changes the regulation of hypoxia-mediated miRNAs. We quantified the abovespecified hypoxia-regulated miRNAs and longitudinally monitored them in the circulation of patients with highgrade gliomas who had been treated with bevacizumab, a recombinant humanized monoclonal antibody that blocks angiogenesis by inhibiting VEGF-A. miRNA levels were correlated with clinical parameters for tumor response, including imaging findings and RANO criteria.

\section{Methods \\ Patients}

A total of 120 serum samples were prospectively obtained for total RNA extraction from 28 adult patients who had been diagnosed with either GBM $(n=23)$ or anaplastic astrocytoma $(n=5$; Table 1$)$. These patients were treated in the Department of Neurosurgery at The Royal Melbourne Hospital or in the Neuro-Oncology Center of Hadassah Hebrew University Medical Center. The study was approved by the local institutional research ethics committee, and all patients signed written consent forms. Eligible patients were treated according to standard or experimental protocols and received first-line temozolomide (TMZ) treatment (standard treatment, TMZ group) or bevacizumab (10 mg/kg every 14 days) either as a second-line treatment at tumor progression or as a first-line therapy as part of the Avaglio study ${ }^{7}$ (clinical trial number NCT00943826; bevacizumab group). The first blood sample $(n=28)$ was obtained prior to the initial diagnostic procedure (surgery or biopsy) and prior to any treatment. All subsequent samples $(n=92)$ were attained within 14 days of a routine follow-up MRI evaluation. In the bevacizumab group, a single sample was taken prior to initiation of bevacizumab treatment, and all subsequent samples were taken during active bevacizumab therapy. At each time point patients were assessed by physical and neurological exam, and tumor responses were evaluated using the RANO criteria. ${ }^{36}$ Routine MRI evaluation included gadolinium-enhanced T1-weighted images and FLAIR sequences that allowed for tumor measurements. The sum of the products of the perpendicular diameters of enhancing lesions and of FLAIR changes were recorded at each clinic visit.

\section{RNA Isolation}

Total RNA was extracted from serum using MasterPure RNA Purification Kit (Epicentre), according to the manufacturer's instructions with the following modifications: prior to RNA extraction, the serum was processed in a centrifuge at room temperature for 1 minute at $14,000 \mathrm{~g}$ to precipitate fat, which might interfere with the reaction, and $1 \mu \mathrm{l}$ of glycogen was added as a carrier at the start of the reaction.

\section{cDNA}

cDNA was produced using the qScript microRNA cDNA Synthesis Kit (Quanta Biosciences), according to the manufacturer's instructions. Briefly, RNA was polyadenylated with ATP by poly(A) polymerase at $37^{\circ} \mathrm{C}$ for 1 hour and then reverse transcribed.

\section{Real-Time Polymerase Chain Reaction Amplification and Relative Quantification}

The miRNA amplification was carried out using primers for the relevant miRNAs, and forward primer sequences appear below. Since the RNA was polyadenylated, oligo $\mathrm{dT}$ was used as the reverse primer.

RNU6-1: GUGCUCGCUUCGGCAGCACAUAUACUA AAAUUGGAACGAUACAGAGAAGAUUAGCAUG 
TABLE 1. Patient characteristics

\begin{tabular}{|c|c|c|}
\hline Characteristic & TMZ Group & $\begin{array}{l}\text { Bevacizumab } \\
\text { Group }\end{array}$ \\
\hline No. of patients & 13 & 15 \\
\hline Median age in yrs (range) & $60(32-78)$ & $54(23-72)$ \\
\hline Sex: M/F & $8 / 5$ & $8 / 7$ \\
\hline Surgical procedure: resection/biopsy & $12 / 1$ & $14 / 1$ \\
\hline \multicolumn{3}{|l|}{ Histology } \\
\hline Anaplastic astrocytoma & 2 & 3 \\
\hline Glioblastoma & 11 & 12 \\
\hline Antiepileptic drugs: $\mathrm{Y} / \mathrm{N}$ & $10 / 3$ & $11 / 4$ \\
\hline \multicolumn{3}{|l|}{ Contrast enhancement } \\
\hline Prior to surgery & 12 & 13 \\
\hline Prior to bevacizumab & 0 & 14 \\
\hline \multicolumn{3}{|l|}{$\begin{array}{l}\text { Median perpendicular diameters of } \\
\text { enhancing lesion(s) in mm (range) }\end{array}$} \\
\hline Prior to surgery & $68(0-152)$ & $48(0-111)$ \\
\hline Prior to bevacizumab & 0 & $87(0-123)$ \\
\hline Last FU & $75(25-182)$ & $82(10-157)$ \\
\hline \multicolumn{3}{|l|}{ Initial therapy } \\
\hline Radiotherapy plus TMZ & 13 & 14 \\
\hline No radiotherapy & 0 & 1 \\
\hline $\begin{array}{l}\text { Median no. of FU serum samples } \\
\text { (range) }\end{array}$ & $4(2-6)$ & $3.5(2-6)$ \\
\hline \multicolumn{3}{|l|}{ Bevacizumab } \\
\hline First-line (Avaglio study) & & 4 \\
\hline Second-line (at progression) & & 11 \\
\hline Median no. of treatments (range) & & $12(2-30)$ \\
\hline \multicolumn{3}{|l|}{$\begin{array}{l}\text { Second-line chemotherapy (w/ beva- } \\
\text { cizumab) }\end{array}$} \\
\hline Carboplatin & & 3 \\
\hline PCV & & 1 \\
\hline None & & 11 \\
\hline
\end{tabular}

$\mathrm{FU}=$ follow-up; $\mathrm{N}=\mathrm{no} ; \mathrm{PCV}=$ procarbazine, $\mathrm{CCNU}$, and vincristine; $\mathrm{Y}=$ yes.

\section{GCCCCUGCGCAAGGAUGACACGCAAAUUCG UGAAGCGUUCCAUAUUUU;} hsa-miR-210-3p: CUGUGCGUGUGACAGCGGCUGA hsa-miR-196b-5p: UAGGUAGUUUCCUGUUGUUGGG hsa-miR-10b-5p: UACCCUGUAGAACCGAAUUUGUG hsa-miR-21-5p: UAGCUUAUCAGACUGAUGUUGA

The reaction was performed using PerfeCTa SYBR Green FastMix ROX (Quanta Biosciences). We selected this technique for quantifying miRNA based on its high performance in terms of reproducibility and accuracy when compared with other methods. ${ }^{23}$ Quantitative polymerase chain reaction (PCR) reactions were done using StepOnePlus real-time PCR (Applied Biosystems) in 96well plates. PCR was initiated at $95^{\circ} \mathrm{C}$ for 2 minutes, followed by 40 cycles at $95^{\circ} \mathrm{C}$ for 5 seconds and $60^{\circ} \mathrm{C}$ for 30 seconds. The specificity of the reaction was verified by melt-curve analysis. The relative quantification (RQ) for each miRNA was calculated based on its average quantification in the serum of 10 healthy volunteers and normalized to RNU6 using the comparative cycle threshold $\left(\mathrm{C}_{\mathrm{T}}\right.$; the cycle number at which the fluorescence signal associated with a particular amplicon accumulation crosses the threshold is referred to as the $\mathrm{C}_{\mathrm{T}}$ ) method (also referred to as the $2^{- \text {DeltaDeltact }}$ method). To analyze the differential quantification between healthy controls and patients with glioma (Fig. 1), RQ has been performed using CT values normalized to RNU6, which is expressed as $2^{-\mathrm{IC}_{\mathrm{T}}(\mathrm{miRNA})}-$ $\mathrm{C}_{\mathrm{T}}{ }^{(\mathrm{RNU} 6]}=\left(2_{\mathrm{T}}^{-\Delta \mathrm{C}}\right)$.

\section{Statistical Methods}

The average fold change in miRNA levels was used to evaluate the quantification level. Quantification levels were compared between healthy controls and tumorbearing patients using the Student t-test. To analyze the association between 2 selected variables (i.e., tumor measurements and miRNA expression or RANO assessment), Pearson correlation coefficients were used based on a 2-tailed test. For analysis involving RANO assessment, disease state was categorized numerically as progressive disease, stable disease, partial response, or complete response. Correlation tests were performed using a binary variable of the fold changes. A p value $<0.05$ was considered statistically significant.

\section{Results}

In a previous study ${ }^{19}$ we surveyed the expression of miRNAs in glial tumors and found 6 miRNAs (miR-302b3p, miR-21, miR-210, miR-10b, miR-196b, and miR-1271) to be highly upregulated in glial tumors as compared with normal brain tissue. The fold increase in expression for these miRNAs in tumors was 3.56, 9.77, 57.02, 107.6, 548, and 1138 for miR-302b-3p, miR-21, miR-210, miR10b, miR-196b, and miR-1271, respectively. As a first step in this study, we evaluated whether these miRNAs could be detected in the serum of healthy individuals and if the increased expression in tumor tissue was reflected in the serum of patients harboring high-grade glioma. miRNA levels were quantified in serum samples from 10 healthy controls and 30 patients with high-grade glioma. Serum from patients with brain tumors was obtained on the day of hospitalization prior to the first diagnostic surgical procedure. Significantly increased levels of miR-10b and miR-21 were detected in the serum of affected patients, while miR210, miR-196b, and miR-1271 levels did not differ from those in healthy controls (Fig. 1). miR-302b-3p was not detected in the serum samples of either healthy controls or patients with brain tumors. Therefore, all further evaluations were performed for miR-10b and miR-21 only.

To assess whether bevacizumab treatment was associated with changing levels of circulating miRNA, we compared findings in the bevacizumab-treated group with those obtained in the TMZ group. The 2 treatment groups did not differ significantly in patient characteristics and tumor sizes, as measured during the initial evaluation and at the last follow-up (Table 1). To evaluate the measurement dynamics at each time point, values were expressed as a fold change from the previous measurement. Each treatment group was assessed separately. Longitudinal evaluations and their association with tumor measurements and RANO results in response to treatment are presented in Table 2. Results for the bevacizumab arm, which illustrates the fold change from pre-bevacizumab values and includes only patients who had at least 2 follow-up evalua- 

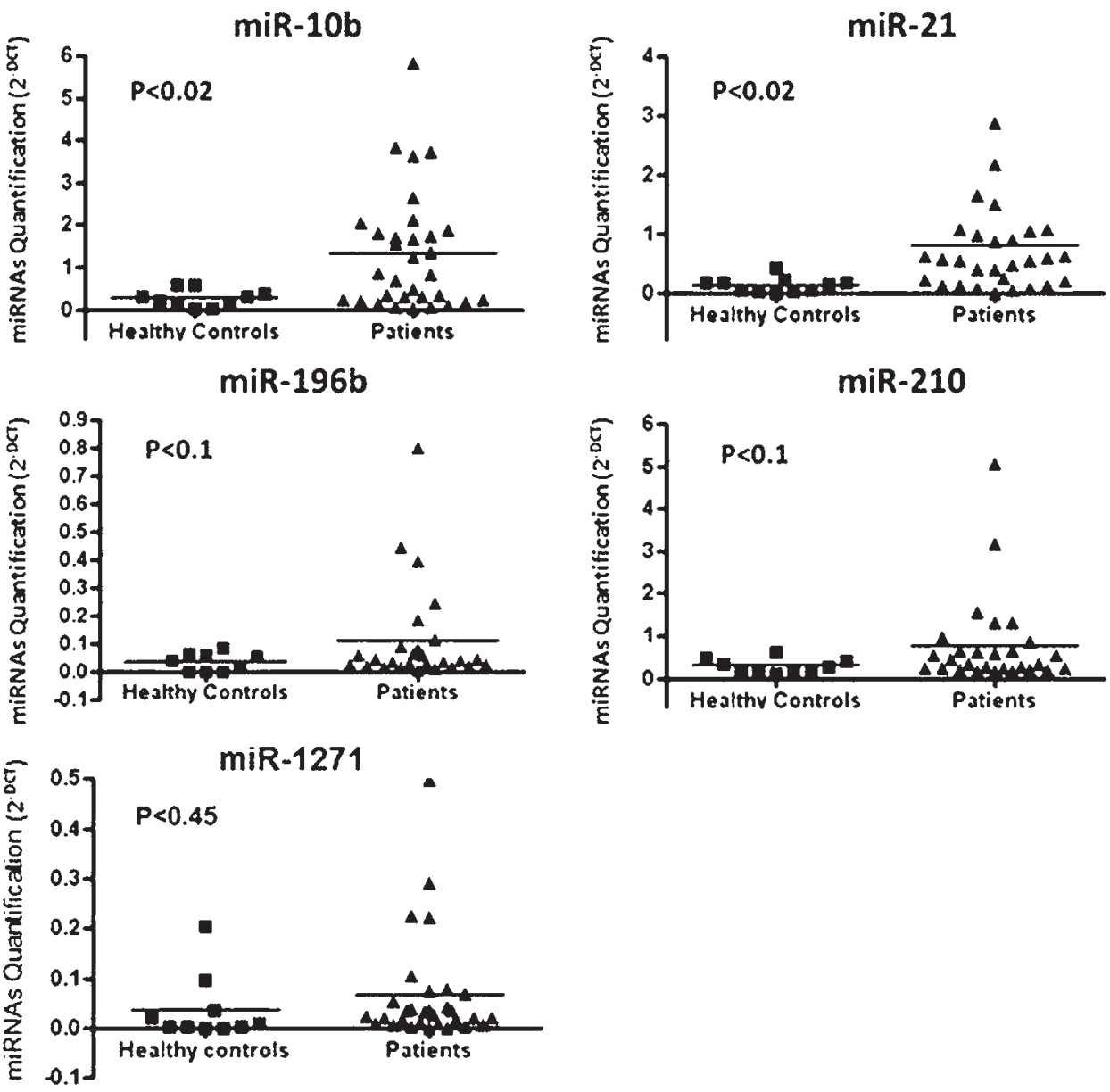

FIG. 1. Circulating hypoxia-regulated miRNA in patients with high-grade glioma and in healthy controls. The scatterplots show the expression of hypoxia-regulated miRNA in serum samples of healthy controls $(n=10)$ and patients with brain tumors $(n=30)$. Serum samples were obtained from patients with high-grade glioma prior to their diagnostic surgical procedure. Analysis was performed by real-time PCR.

tions on bevacizumab treatment, are shown in Figs. 2 and 3. Both miR-10b and miR-21 tend to display high expression levels when compared with pre-bevacizumab levels, as demonstrated in Fig. 2 upper, which shows the sum of the relative quantification of the 2 miRNAs for each time point. The sum quantification is based on previous studies showing that a combination of miRNAs yielded better diagnostic results than individual miRNAs alone. . $^{2,3,28,29,38}$ In 9 of the 15 patients, miR-10b and miR-21 were highly induced (with fold induction ranging from 1.5 to 18 and from 1.5 to 5 , respectively) following the first bevacizumab treatment. Figure 2 lower shows the longitudinal changes that occurred in the enhancing tumor measurements on MRI. In most patients, the sum of the products of the perpendicular enhancing diameters were clearly reduced with bevacizumab treatment, as expected. However, a reduction of greater than $50 \%$, which is required to be classified as a partial response according to the RANO criteria, was not

TABLE 2. Associations between fold change in miRNA quantification and tumor measurement or RANO assessment in patients treated with TMZ or bevacizumab

\begin{tabular}{|c|c|c|c|c|c|c|}
\hline \multirow[b]{2}{*}{ Parameter } & \multicolumn{2}{|c|}{ miR-10b } & \multicolumn{2}{|c|}{ miR-21 } & \multicolumn{2}{|c|}{ Sum of miR-10b \& miR-21 } \\
\hline & TMZ & Bev & TMZ & Bev & TMZ & Bev \\
\hline Sum of enhancing tumor diameters & $\begin{array}{l}r=-0.023 \\
p=0.906\end{array}$ & $\begin{array}{l}r=-0.51 \\
p<0.002\end{array}$ & $\begin{array}{l}r=-0.106 \\
p=0.532\end{array}$ & $\begin{array}{l}r=-0.568 \\
p<0.0001\end{array}$ & $\begin{array}{l}r=0.071 \\
p=0.676\end{array}$ & $\begin{array}{l}r=-0.648 \\
p<0.0001\end{array}$ \\
\hline Sum of FLAIR diameters & $\begin{array}{l}r=-0.249 \\
p=0.137\end{array}$ & $\begin{array}{l}r=-0.365 \\
p=0.031\end{array}$ & $\begin{array}{l}r=-0.173 \\
p=0.314\end{array}$ & $\begin{array}{l}r=-0.248 \\
p=0.151\end{array}$ & $\begin{array}{l}r=-0.094 \\
p=0.580\end{array}$ & $\begin{array}{l}r=-0.322 \\
p=0.059\end{array}$ \\
\hline RANO classification & $\begin{array}{l}r=-0.08 \\
p=0.703\end{array}$ & $\begin{array}{l}r=-0.309 \\
p=0.068\end{array}$ & $\begin{array}{l}r=-0.32 \\
p=0.118\end{array}$ & $\begin{array}{l}r=-0.189 \\
p=0.270\end{array}$ & $\begin{array}{l}r=-0.161 \\
p=0.441\end{array}$ & $\begin{array}{l}r=-0.116 \\
p=0.523\end{array}$ \\
\hline
\end{tabular}

Bev = bevacizumab-treated group; TMZ = TMZ-treated group. 

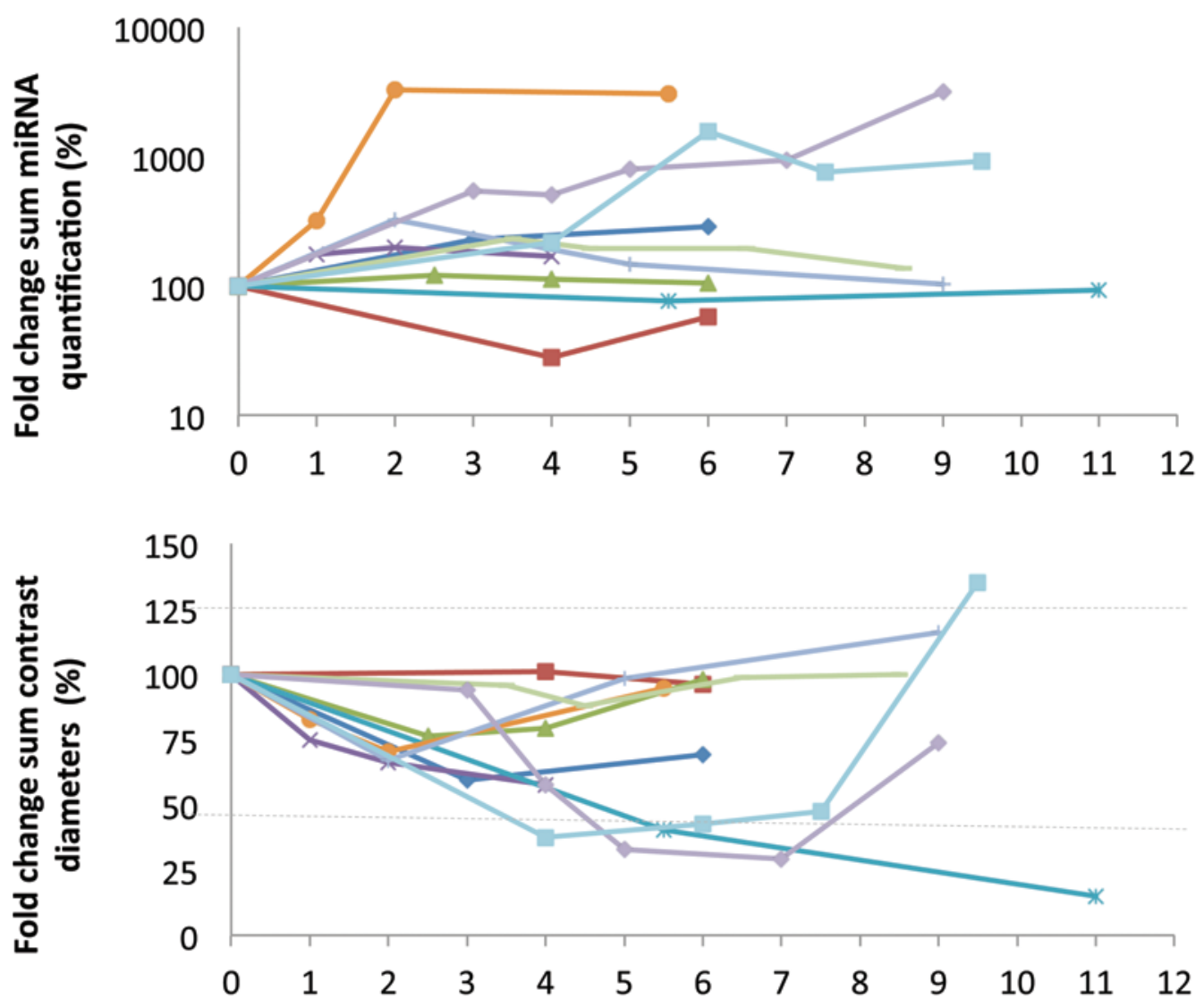

Months on Bevacizumab

FIG. 2. Dynamics of circulating miRNA expression and enhancing tumor diameters during bevacizumab treatment. Fold change in the sum over time is shown for circulating miR-10b and miR-21 levels (upper) and for cross-sectional enhancing diameters of the tumor measured using enhanced T1-weighted MR images (lower). Each patient is represented by a separate line and color that identifies them in both figures. Only patients with more than 2 follow-up serum samples during treatment are represented in this figure.

common. At each time point, response to treatment was also assessed by the RANO criteria, and the longitudinal results are shown in Fig. 3. It is clear that the combined clinicoradiological assessment classified most patients as having stable disease prior to the point at which progressive disease was observed, and some patients maintained the response to treatment for a prolonged period of time.

Because the assessed variables (cross-sectional FLAIR, enhancing tumor diameters, and miR-10b and miR-21 levels) are all swayed by bevacizumab treatment and possibly by steroid treatment, their causal sequence remains unclear. Therefore, to assess whether changes in circulating miRNA levels reflect tumor response to bevacizumab therapy or to chemotherapy, the fold change for each variable at each time point was used in the correlation analysis, which was performed for both the TMZ group and the bevacizumab group.

Table 2 presents the correlations between fold change in miRNA expression levels and the radiological evaluation of tumor dimensions or changes in tumor response as assessed by RANO criteria. A significant negative correlation was found only in the bevacizumab group between enhancing tumor diameters and fold change for either
miRNA alone or the average change for both miRNAs combined. On the contrary, changes in FLAIR measurements in the bevacizumab group were found to correlate significantly and negatively only with fold change in miR$10 \mathrm{~b}$ levels, and no significant correlation was found between changes in RANO classification and the expression of miRNAs. In the TMZ group, no significant correlation was found between the fold change in miRNA levels (either alone or combined) and tumor dimension or clinical response.

\section{Discussion}

This study reveals the dynamic effects of antiangiogenic therapy on levels of circulating miR-10b and miR21 in patients with high-grade gliomas. Levels of both miRNAs are markedly altered by bevacizumab treatment, and this increase tends to persist throughout the treatment period. To our knowledge, no previous prospective longitudinal monitoring of circulating miRNA has been published to date in patients with brain tumors. Furthermore, there are no other studies that prospectively explore whether these biomarkers reflect tumor burden, 


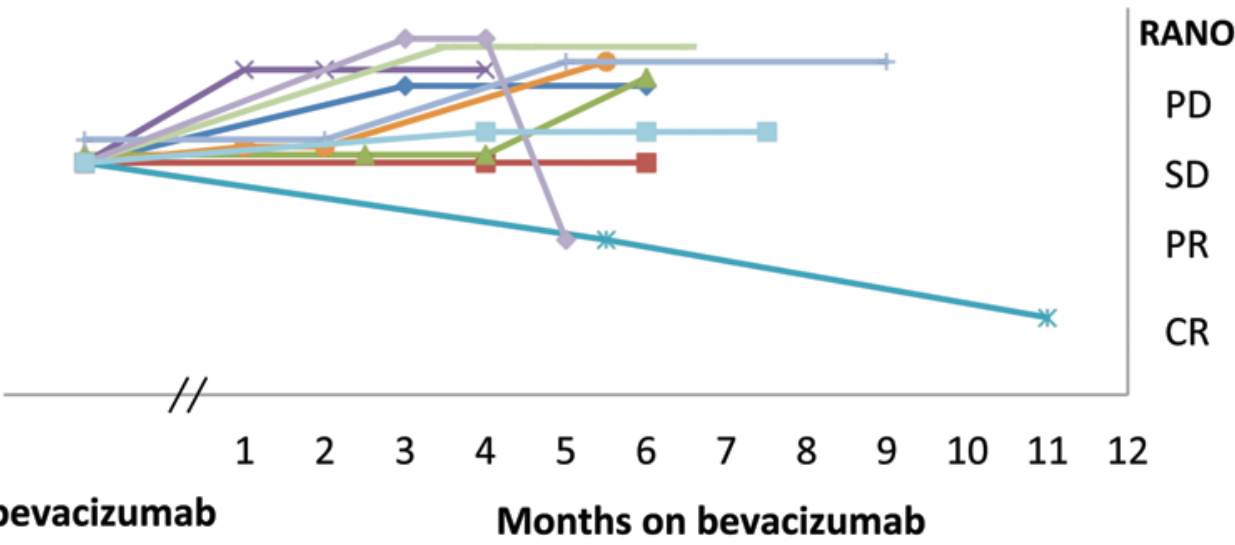

FIG. 3. Assessment of tumor response during bevacizumab treatment using RANO criteria. RANO assessment was performed within 14 days of the MRI study. Each patient is represented by a separate line and color. Only patients with more than 2 follow-up serum samples during treatment are represented in this figure. $C R=$ complete response; $P D=$ progressive disease; $P R=$ partial response; $\mathrm{SD}=$ stable disease.

as assessed by clinical and imaging measures. Circulating tumor-specific miRNAs have been detected in the blood of patients with GBM in 4 studies. ${ }^{9,11,29,35,39}$ These studies found increased levels of miR-21, miR-128, ${ }^{29,35} \mathrm{miR}-20 \mathrm{a}-$ 5p, miR-106a-5p, and miR-181b-5p. ${ }^{39}$ Elevated circulating miR-21 was incidentally detected 36 months prior to the diagnosis of GBM in a single patient who participated in a prospective study of Alzheimer's disease. ${ }^{11}$ One study compared the blood profile of 1158 miRNAs in 20 patients with GBM and 20 age-matched healthy controls and found that only 2 candidates, miR-128 and miR342$3 p$, remained significantly elevated after correcting for multiple testing. ${ }^{29}$

Any tumor-derived marker is expected to be at its highest concentration in the tumor itself, followed by the surrounding extracellular fluid and then the peripheral blood. Cerebrospinal fluid analysis may serve as a close reflection of the tumor's extracellular space, but unfortunately this is not practical in patients with high-grade glioma. One study attempted to distinguish between GBM and brain metastases (primary tumors from breast and lung) through miRNA profiling of patients' CSF. ${ }^{32}$ The analysis revealed an elevation in miR-10b and miR-21 levels for all categories of brain neoplasms, as compared with tumors in remission or with a variety of nonneoplastic conditions. In the current study, our search for circulating markers was based on our previous findings in tumor tissues in which both miR$10 \mathrm{~b}$ and miR-21 were found to be highly expressed in tumors as compared with normal brains. ${ }^{19}$ However, aberrant expression of miRNAs in neural tissue or in a systemic tumor is not always reflected in the circulation, as was encountered in other studies. ${ }^{14,20,37}$ Indeed, we also found that circulating levels of miR-210, miR-196b, and miR-1271 in patients with brain tumors was similar to levels in healthy adults, despite the fact that their fold expression in tumor tissue was 57.02, 548, and 1138, respectively. That left us with 2 hypoxia-regulated markers, miR-10b and miR-21, that were significantly elevated in the bloodstream of patients with high-grade glioma-and according to previous findings, also in glial tumors. ${ }^{19}$

miRNAs are supposed to enter body fluids via 3 primary mechanisms: 1) passive release from normal or dis- eased tissue, 2) active secretion from cell-born membrane vesicles (exosomes, microvesicles, and apoptotic bodies), and 3) active secretion by macromolecular complexes (e.g., high density lipoprotein). ${ }^{27}$ The cellular origin of bloodborne miRNA is not fully explored, but primary sources could include blood cells (red and white blood cells), platelets, ${ }^{26}$ vascular endothelium, liver, and spleen. ${ }^{6}$ The global relationship between tissue miRNA and circulating miRNA in the normal population has been investigated, ${ }^{6,13,21}$ and a significant positive correlation has been observed between blood-borne miRNA and tissue-borne miRNA. Liver miRNA correlates most closely with circulating miRNA, while miRNA from placenta, testis, and brain exhibits the weakest correlation. ${ }^{6}$ Although a positive correlation between tissue and blood miRNA is observed in the normal population, the relationship between cancer tissue and blood samples is more complex. A pattern opposite to what is expected, based on tissue profile, has been observed in the circulation of cancer patients for deregulated tumor-suppressing miRNA and oncogenic miRNA levels. ${ }^{13,22}$ Thus, the cause of altered miR-10b and miR-21 levels, which are observed in our patients during bevacizumab treatment, remains unclear, as both the highgrade glioma and systemic tissues could be contributing sources. Given that both miRNAs were elevated throughout the treatment period (Fig. 2 upper), it is implied that the anti-VEGF treatment affects the regulation of these 2 hypoxia-regulated miRNAs, probably as a compensatory mechanism to overcome the antiangiogenic effect of bevacizumab. Of note are 2 previous studies that evaluated miRNA expression in patients treated with bevacizumab. ${ }^{12,34}$ One study used biopsies of metastatic melanomas obtained prior to the start of treatment and during therapy. ${ }^{34}$ It showed that tumor expression of 15 miRNAs, including miR-10b, was significantly upregulated once bevacizumab was included in the treatment regimen. The second study ${ }^{12}$ assessed circulating miRNA levels in patients with non-small cell lung cancer 24 hours after the first dose of a treatment regimen that included bevacizumab. It found no significant change from the pretreatment profile. However, this 24-hour time point may be too early to detect a significant change in circulating markers. 
The longitudinal assessment conducted during bevacizumab treatment was meant to evaluate whether circulating miRNA levels may reflect changes in tumor burden over time by using correlative imaging and clinical data at each time point. The significant negative correlation between miR-10b and miR-21 levels and cross-sectional enhancing tumor diameters (Table 2) is not surprising if we assume that their upregulation reflects the effect of antiangiogenic therapy (Fig. 2). The dramatic reduction in the amount of contrast enhancement has been previously noted as an early effect of antiangiogenic therapy, ${ }^{1,4,16,25}$ but it is becoming increasingly recognized that changes in vascular permeability may be independent of the antitumor effect. ${ }^{10}$ For that reason, we also used FLAIR images to investigate nonenhancing tumor and used RANO criteria to assess tumor response. With the current lack of specific guidelines, ${ }^{8}$ we are well aware of the concerns regarding reproducibility in the determination of nonenhancing tumor progression, but it seems that the FLAIR measurements further reflect the limited effect of bevacizumab treatment on tumor burden in this study. For the most part, both RANO assessment and FLAIR findings suggest that tumors probably stabilized with bevacizumab treatment, but there was a lack of consistent correlation between tumor status and miRNA levels. The sum quantification of both miRNAs remained elevated and relatively stable at the point where progressive disease was noted by RANO assessment (Figs. 2 upper and 3). Finally, the lack of significant association between circulating miRNA, tumor measurements, and RANO assessment in the TMZ group (Table 2) further suggests that the increased circulating miRNA levels observed with bevacizumab are a result of the antiangiogenic effect of the treatment.

The main shortcoming of our study is the small number of patients, despite the fact that 120 blood samples were collected prospectively and matched with clinical and imaging parameters. Future studies should include larger cohorts of patients treated with different modalities (such as chemotherapy, antiangiogenic therapy, or immunotherapy) to verify the clinical utility of using circulating miRNA as a biomarker.

\section{Conclusions}

This study demonstrates that circulating miR-10b and miR-21 tend to be present at high levels during bevacizumab treatment, which probably reflects the antiangiogenic effect of therapy. Their role as biomarkers for tumor response remains uncertain and requires further investigation using a larger-scale study.

\section{Acknowledgments}

We thank Lucy Paradiso and Professor Kate J. Drummond from the Departments of Neurosurgery and Surgery, The Royal Melbourne Hospital and the University of Melbourne, for laboratory technical assistance and for clinical help and support related to patient management; Dr. Yigal Shoshan from the Department of Neurosurgery and Anat Mordechai and Anat Bar-Halevi from the Gaffin Center for Neuro-Oncology, Hadassah University Hospital, for their help in patient care and management, data management, and study coordination; and Neal Tsur from Hebrew University for assistance with statistics and graphic presentations. We also thank the Israel Cancer Association for the generous assistance of the ICA USA 2011 Golf Classic Proceeds, The Royal Melbourne Hospital Neuroscience Foundation, and the Brain Foundation of Australia for supporting this work.

\section{References}

1. Ananthnarayan S, Bahng J, Roring J, Nghiemphu P, Lai A, Cloughesy T, et al: Time course of imaging changes of GBM during extended bevacizumab treatment. J Neurooncol 88:339-347, 2008

2. Baraniskin A, Kuhnhenn J, Schlegel U, Chan A, Deckert M, Gold R, et al: Identification of microRNAs in the cerebrospinal fluid as marker for primary diffuse large B-cell lymphoma of the central nervous system. Blood 117:3140-3146, 2011

3. Baraniskin A, Kuhnhenn J, Schlegel U, Maghnouj A, Zöllner $\mathrm{H}$, Schmiegel W, et al: Identification of microRNAs in the cerebrospinal fluid as biomarker for the diagnosis of glioma. Neuro Oncol 14:29-33, 2012

4. Batchelor TT, Sorensen AG, di Tomaso E, Zhang WT, Duda DG, Cohen KS, et al: AZD2171, a pan-VEGF receptor tyrosine kinase inhibitor, normalizes tumor vasculature and alleviates edema in glioblastoma patients. Cancer Cell 11:83-95, 2007

5. Bergers G, Hanahan D: Modes of resistance to anti-angiogenic therapy. Nat Rev Cancer 8:592-603, 2008

6. Chen G, Wang J, Cui Q: Could circulating miRNAs contribute to cancer therapy? Trends Mol Med 19:71-73, 2013

7. Chinot OL, Wick W, Mason W, Henriksson R, Saran F, Nishikawa R, et al: Bevacizumab plus radiotherapy-temozolomide for newly diagnosed glioblastoma. N Engl J Med 370:709-722, 2014

8. Ellingson BM, Wen PY, van den Bent MJ, Cloughesy TF: Pros and cons of current brain tumor imaging. Neuro Oncol 16 (Suppl 7):vii2-vii11, 2014

9. Holdhoff M, Yovino SG, Boadu O, Grossman SA: Bloodbased biomarkers for malignant gliomas. J Neurooncol 113:345-352, 2013

10. Hygino da Cruz LC Jr, Rodriguez I, Domingues RC, Gasparetto EL, Sorensen AG: Pseudoprogression and pseudoresponse: imaging challenges in the assessment of posttreatment glioma. AJNR Am J Neuroradiol 32:1978-1985, 2011

11. Ilhan-Mutlu A, Wagner L, Wöhrer A, Jungwirth S, Marosi C, Fischer P, et al: Blood alterations preceding clinical manifestation of glioblastoma. Cancer Invest 30:625-629, 2012

12. Joerger M, Baty F, Früh M, Droege C, Stahel RA, Betticher DC, et al: Circulating microRNA profiling in patients with advanced non-squamous NSCLC receiving bevacizumab/erlotinib followed by platinum-based chemotherapy at progression (SAKK 19/05). Lung Cancer 85:306-313, 2014

13. Keller A, Leidinger P, Bauer A, Elsharawy A, Haas J, Backes $\mathrm{C}$, et al: Toward the blood-borne miRNome of human diseases. Nat Methods 8:841-843, 2011

14. Keller A, Leidinger P, Lange J, Borries A, Schroers H, Scheffler M, et al: Multiple sclerosis: microRNA expression profiles accurately differentiate patients with relapsing-remitting disease from healthy controls. PLoS One 4:e7440, 2009

15. Keunen O, Johansson M, Oudin A, Sanzey M, Rahim SA, Fack F, et al: Anti-VEGF treatment reduces blood supply and increases tumor cell invasion in glioblastoma. Proc Natl Acad Sci U S A 108:3749-3754, 2011

16. Kreisl TN, Kim L, Moore K, Duic P, Royce C, Stroud I, et al: Phase II trial of single-agent bevacizumab followed by bevacizumab plus irinotecan at tumor progression in recurrent glioblastoma. J Clin Oncol 27:740-745, 2009

17. Kros JM, Mustafa DM, Dekker LJ, Sillevis Smitt PA, Luider TM, Zheng PP: Circulating glioma biomarkers. Neuro Oncol 17:343-360, 2015

18. Lavon I, Refael M, Zelikovitch B, Shalom E, Siegal T: Serum 
DNA can define tumor-specific genetic and epigenetic markers in gliomas of various grades. Neuro Oncol 12:173-180, 2010

19. Lavon I, Zrihan D, Granit A, Einstein O, Fainstein N, Cohen MA, et al: Gliomas display a microRNA expression profile reminiscent of neural precursor cells. Neuro Oncol 12:422433, 2010

20. Leidinger P, Keller A, Borries A, Reichrath J, Rass K, Jager SU, et al: High-throughput miRNA profiling of human melanoma blood samples. BMC Cancer 10:262, 2010

21. Liang Y, Ridzon D, Wong L, Chen C: Characterization of microRNA expression profiles in normal human tissues. BMC Genomics 8: 166, 2007

22. Lu M, Shi B, Wang J, Cao Q, Cui Q: TAM: a method for enrichment and depletion analysis of a microRNA category in a list of microRNAs. BMC Bioinformatics 11:419, 2010

23. Mestdagh P, Hartmann N, Baeriswyl L, Andreasen D, Bernard N, Chen C, et al: Evaluation of quantitative miRNA expression platforms in the microRNA quality control (miRQC) study. Nat Methods 11:809-815, 2014

24. Pàez-Ribes M, Allen E, Hudock J, Takeda T, Okuyama H, Viñals F, et al: Antiangiogenic therapy elicits malignant progression of tumors to increased local invasion and distant metastasis. Cancer Cell 15:220-231, 2009

25. Pope WB, Lai A, Nghiemphu P, Mischel P, Cloughesy TF: MRI in patients with high-grade gliomas treated with bevacizumab and chemotherapy. Neurology 66:1258-1260, 2006

26. Pritchard CC, Kroh E, Wood B, Arroyo JD, Dougherty KJ, Miyaji MM, et al: Blood cell origin of circulating microRNAs: a cautionary note for cancer biomarker studies. Cancer Prev Res (Phila) 5:492-497, 2012

27. Redis RS, Calin S, Yang Y, You MJ, Calin GA: Cell-to-cell miRNA transfer: from body homeostasis to therapy. Pharmacol Ther 136:169-174, 2012

28. Roth P, Keller A, Hoheisel JD, Codo P, Bauer AS, Backes $\mathrm{C}$, et al: Differentially regulated miRNAs as prognostic biomarkers in the blood of primary CNS lymphoma patients. Eur J Cancer 51:382-390, 2015

29. Roth P, Wischhusen J, Happold C, Chandran PA, Hofer S, Eisele G, et al: A specific miRNA signature in the peripheral blood of glioblastoma patients. J Neurochem 118:449-457, 2011

30. Rubenstein JL, Kim J, Ozawa T, Zhang M, Westphal M, Deen DF, et al: Anti-VEGF antibody treatment of glioblastoma prolongs survival but results in increased vascular cooption. Neoplasia 2:306-314, 2000

31. Shen G, Li X, Jia YF, Piazza GA, Xi Y: Hypoxia-regulated microRNAs in human cancer. Acta Pharmacol Sin 34:336341, 2013

32. Teplyuk NM, Mollenhauer B, Gabriely G, Giese A, Kim E, Smolsky M, et al: MicroRNAs in cerebrospinal fluid identify glioblastoma and metastatic brain cancers and reflect disease activity. Neuro Oncol 14:689-700, 2012
33. Thompson EM, Frenkel EP, Neuwelt EA: The paradoxical effect of bevacizumab in the therapy of malignant gliomas. Neurology 76:87-93, 2011

34. Wagenseller AG, Shada A, D'Auria KM, Murphy C, Sun $\mathrm{D}$, Molhoek KR, et al: MicroRNAs induced in melanoma treated with combination targeted therapy of Temsirolimus and Bevacizumab. J Transl Med 11:218, 2013

35. Wang Q, Li P, Li A, Jiang W, Wang H, Wang J, et al: Plasma specific miRNAs as predictive biomarkers for diagnosis and prognosis of glioma. J Exp Clin Cancer Res 31:97, 2012

36. Wen PY, Macdonald DR, Reardon DA, Cloughesy TF, Sorensen AG, Galanis E, et al: Updated response assessment criteria for high-grade gliomas: response assessment in neuro-oncology working group. J Clin Oncol 28:1963-1972, 2010

37. Witwer KW: Circulating microRNA biomarker studies: pitfalls and potential solutions. Clin Chem 61:56-63, 2015

38. Yang C, Wang C, Chen X, Chen S, Zhang Y, Zhi F, et al: Identification of seven serum microRNAs from a genomewide serum microRNA expression profile as potential noninvasive biomarkers for malignant astrocytomas. Int J Cancer 132:116-127, 2013

39. Zhi F, Shao N, Wang R, Deng D, Xue L, Wang Q, et al: Identification of 9 serum microRNAs as potential noninvasive biomarkers of human astrocytoma. Neuro Oncol 17:383391, 2015

\section{Disclosures}

The authors report no conflict of interest concerning the materials or methods used in this study or the findings specified in this paper.

\section{Author Contributions}

Conception and design: Kaye, Siegal, Lavon. Acquisition of data: Kaye, Siegal, Charbit, Paldor, Zelikovitch, Canello, Wong, Morokoff, Lavon. Analysis and interpretation of data: Siegal, Charbit, Paldor, Lavon. Drafting the article: Siegal, Lavon. Critically revising the article: Kaye, Siegal, Morokoff, Lavon. Reviewed submitted version of manuscript: all authors. Statistical analysis: Benis. Administrative/technical/material support: Kaye, Siegal, Charbit, Wong, Lavon. Study supervision: Siegal.

\section{Correspondence}

Andrew Kaye, Department of Surgery, The University of Melbourne, 6th Fl., Center for Medical Research, The Royal Melbourne Hospital, Parkville, Victoria 3050, Australia. email: a.kaye@unimelb.edu.au. 